\title{
An Oligonucleotide Array for the Identification and Differentiation of Bacteria Pathogenic on Potato
}

\author{
Anania Fessehaie, Solke H. De Boer, and C. André Lévesque
}

First and third authors: Eastern Cereal and Oilseed Research Centre, Research Branch, Agriculture and Agri-Food Canada, 960 Carling Avenue, Ottawa, Ontario K1A 0C6; and second author: Canadian Food Inspection Agency, Centre for Animal and Plant Health, 93 Mount Edward Road, Charlottetown, Prince Edward Island C1A 5T1. Accepted for publication 2 October 2002.

\begin{abstract}
Fessehaie, A., De Boer, S. H., and Lévesque, C. A. 2003. An oligonucleotide array for the identification and differentiation of bacteria pathogenic on potato. Phytopathology 93:262-269.

Oligonucleotides, 16 to 24 bases long, were selected from the $3^{\prime}$ end of the $16 \mathrm{~S}$ gene and the $16 \mathrm{~S}-23 \mathrm{~S}$ intergenic spacer regions of bacteria pathogenic on potato, including Clavibacter michiganensis subsp. sepedonicus, Ralstonia solanacearum, and the pectolytic erwinias, including Erwinia carotovora subsp. atroseptica and carotovora and E. chrysanthemi. Oligonucleotides were designed and formatted into an array by pin

digoxigenin label revealed different hybridization patterns that were distinct for each species and subspecies tested. Hybridization of amplicons generally was restricted to appropriate homologous oligonucleotides and cross-hybridization with heterologous oligonucleotides was rare. Hybridization patterns were recorded as separate gray values for each hybridized spot and revealed a consistent pattern for multiple strains of each species or subspecies isolated from diverse geographical regions. In preliminary tests, bacteria could be correctly identified and detected by hybridizing to the array amplicons from mixed cultures and inoculated potato tissue.
\end{abstract} spotting on nylon membranes. Genomic DNA from bacterial cultures was amplified by polymerase chain reaction using conserved ribosomal primers and labeled simultaneously with digoxigenin-dUTP. Hybridization of amplicons to the array and subsequent serological detection of
Additional keywords: blackleg, brown rot, macroarray, microarray, reverse dot blot, ring rot, soft rot.
The gram-positive bacterium, Clavibacter michiganensis subsp. sepedonicus, and gram-negative bacteria, Ralstonia solanacearum and pectolytic erwinias, including Erwinia carotovora subsp. atroseptica and carotovora and E. chrysanthemi, are important bacterial pathogens of potato. E. carotovora subsp. carotovora is ubiquitous in most agricultural environments, surviving in surface water and soil, often in association with decaying crop residues (29). Although bacterial potato pathogens contaminate healthy seed potatoes from infected crop residues and other inoculum sources, the main cause of disease is seed potato tubers that were contaminated with the pathogen during the previous growing season (3). Often, contamination or infection is at a subclinical level, in that no overt symptoms of disease are present. Such latent infections cannot be detected by visual inspection of tuber lots. Consequently, laboratory tests are used to detect C. michiganensis subsp. sepedonicus and $R$. solanacearum in seed tuber lots to control, respectively, bacterial ring rot disease in Canada and brown rot in western Europe $(4,14)$. Tests for E. carotovora subsp. atroseptica and E. chrysanthemi also have been developed, but these have been used to a lesser extent due to the high cost of laboratory testing and the lesser importance of the diseases they incite (28).

The conventional approach to testing for latent bacterial infections in seed potato has been to use serological methods such as enzyme-linked immunosorbent assay and immunofluorescence, sometimes coupled with bioassay or isolation procedures $(4,8,28)$.

Corresponding author: C. A. Lévesque; E-mail address: LevesqueCA@agr.gc.ca

Publication no. P-2003-0114-01R

This article is in the public domain and not copyrightable. It may be freely reprinted with customary crediting of the source. The American Phytopathological Society, 2003.
With the development of species-specific oligonucleotide primers, polymerase chain reaction (PCR) now also can be used as a confirmatory assay. Although the sensitivity of PCR may make its use advantageous, particularly if conducted with one of the realtime technologies (30) which alleviates the need for postamplification identification of PCR product, its widespread use for the potato industry is prohibited by its cost and intensive labor requirements.

DNA array technology, essentially a reverse dot blot technique, is an emerging methodology useful for identification of DNA fragments and may be applicable for rapid identification and detection of plant pathogens associated with plants $(20,21,26)$. An array of species-specific oligonucleotide probes representing the various pathogens of potato, built on a solid support such as a nylon membrane or microscope slide, could be probed readily with labeled PCR products amplified from a potato sample. By using conserved primers to amplify common bacterial genome fragments from extracts of potato tubers that might contain the bacterial pathogens, the presence of DNA sequences indicative of pathogenic species would be revealed by hybridization to speciesspecific oligonucleotide probes within the array.

This strategy has been used elsewhere. For example, a nonradioactive reverse dot blot method was used initially for the detection of tickborne encephalitis in clinical specimens (11) and, subsequently, for the diagnosis of point mutations in congenital adrenal hyperplasia caused by 21-hydroxylase deficiency (37), and was particularly useful for the identification of retroviral sequences (31). A similar strategy was used to identify lactic acid bacteria in fermented food by targeting specific genomic sequences in the ribosomal gene cluster (7), as well as for diagnosis of Staphylococcus spp. by targeting marker genes (13). In plant pathology, array technology targeting the ribosomal gene cluster also was successfully applied to identify oomycete fungi (22) and nematodes (34). 
In this study, we explored the feasibility of using DNA array technology for identification and differentiation of plant pathogenic bacteria by targeting five bacterial pathogens of potato. We identified oligonucleotides in the $16 \mathrm{~S}$ and the 16S-23S intergenic spacer (IGS) region of the ribosomal gene clusters of the potato bacterial pathogens that were specific for each of the bacteria. Both pure cultures of bacteria and potato extracts were evaluated on arrays established with the specific and heterologous oligonucleotides to determine the discriminatory potential of this technology and application for identification and detection of bacterial pathogens. A preliminary report on a part of this study has been published (10).

\section{MATERIALS AND METHODS}

Bacterial strains. The bacterial strains used in this study are listed in Table 1. Cells of Erwinia spp. were cultured in tryptone yeast extract liquid media containing $1.6 \%$ (wt/vol) tryptone, $1 \%$ (wt/vol) yeast extract, and $0.5 \%$ (wt/vol) $\mathrm{NaCl}$ (32). Strains of $C$. michiganensis subsp. sepedonicus and $R$. solanacearum were grown at ambient temperature in nutrient broth supplemented with yeast extract (30). Stock bacterial cultures were maintained on the same medium supplemented with $15 \%(\mathrm{wt} / \mathrm{vol})$ glycerol at $-70^{\circ} \mathrm{C}$.
DNA extraction. Cultures of Erwinia spp., C. michiganensis subsp. sepedonicus, and $R$. solanacearum were harvested in late $\log$ phase. Genomic DNA was extracted from 50 to $100 \mathrm{mg}$ fresh weight of cells using a commercial DNA Purification Kit (BIO 101, LaJolla, CA).

PCR amplification, DNA sequencing, and labeling. PCR and sequencing primers were as published in a previous study (9). Amplification and sequencing of $16 \mathrm{~S}$ rRNA genes was performed to confirm the identity of the strains used in this study as described (9). For digoxigenin-labeling of DNA template, PCR reactions were carried out in a GeneAmp PCR Systems 2400 (Applied Biosystems, Foster City, CA) in $25 \mu \mathrm{l}$ with $1 \times \mathrm{Mg}^{++}$-free PCR buffer (Gibco-BRL, Burlington, Ontario, Canada), $2.0 \mathrm{mM}$ $\mathrm{MgCl}_{2}, 0.16 \mathrm{mM}$ dNTPs each, $0.4 \mu \mathrm{M}$ primer each, 2.5 units of Taq DNA polymerase (Gibco/BRL), and $1 \mu \mathrm{l}$ of genomic DNA (10 to $15 \mathrm{ng} / \mu \mathrm{l}$ ) with the following thermal profile: $94^{\circ} \mathrm{C}$ for 2 min followed by 35 cycles of $94^{\circ} \mathrm{C}$ for $45 \mathrm{~s}, 64^{\circ} \mathrm{C}$ for $45 \mathrm{~s}$, and $72^{\circ} \mathrm{C}$ for $1.5 \mathrm{~min}$, and a final extension at $72^{\circ} \mathrm{C}$ for $9 \mathrm{~min}$. Primers 1114f (5'-GCAACGAGCGCAACCCT-3') (19) and L1r (5'CA(A/G)GGCATCCACCGT-3') (12) from conserved sequences flanking the 16S/IGS region were used in PCR to generate digoxigenin-dUTP-labeled amplicons from bacterial DNA templates. Concentration of PCR products was estimated on $1.5 \%$ agarose (OMNI PUR, EMScience, Gibbstown, NJ).

TABLE 1. Strain, host, and source information for the bacterial species studied

\begin{tabular}{|c|c|c|c|}
\hline Bacteria, strains, origin ${ }^{\mathrm{a}}$ & Host & Bacteria, strains, origin ${ }^{a}$ & Host \\
\hline \multicolumn{2}{|l|}{ Erwinia carotovora subsp. atroseptica } & \multicolumn{2}{|l|}{ E. herbicola (P. agglomerans) } \\
\hline UBC E1; B. Copeman, B.C., Canada & Solanum tuberosum & GSPB 450; R. A. Lelliot, England & Pyrus communis \\
\hline 31; A. Kelman (SR8), Wisconsin, USA & S. tuberosum & LMG 2565; E. Roslycky, Canada & Cereals \\
\hline 558; H. Maas Geesteranus, Netherlands & S. tuberosum & GSPB 2791; E. French, Peru & S. tuberosum \\
\hline BBA 9201; F. Niepold, Braunschweig, Germany & S. tuberosum & LMG 2300; Z. Volcani, Israel & S. tuberosum \\
\hline BBA 9204; F. Niepold, Braunschweig, Germany & S. tuberosum & LMG 2305; R. Lelliott, Egypt & S. tuberosum \\
\hline BBA 9205; F. Niepold, Braunschweig, Germany & S. tuberosum & \multicolumn{2}{|l|}{ Rhizoctonia solanacearum } \\
\hline GSPB 2231; S. H. De Boer, B.C., Canada & S. tuberosum & PD 1424; M. Goto, Japan & Arachis hypogea \\
\hline \multicolumn{2}{|l|}{ E. carotovora subsp. odorifera } & PD 2763; J. Janse, The Netherlands & S. tuberosum \\
\hline 1645-1; R. Samson (16454-1), France & Cichorium intybus & GSPB 2695; E. French, Kenya & L. esculentum \\
\hline 1878; R. Samson (1878), France & C. intybus & GSPB 2791; E. French, Peru & S. tuberosum \\
\hline 1880; R. Samson (1880), France & C. intybus & LMG 2300; Z. Volcani, Isreal & S. tuberosum \\
\hline 1893; CFBP 1893/R. Samson, France & C. intybus & LMG 2305; R. Lelliott, Egypt & S. tuberosum \\
\hline \multicolumn{2}{|l|}{ E. carotovora subsp. betavasculorum } & \multicolumn{2}{|l|}{ Pseudomonas fluorescens } \\
\hline SR 94; M. Goto (94), Japan & A. rusticana & CS 3M; G. A. Nelson, Canada & S. tuberosum \\
\hline E. carotovora subsp. carotovora & & CS R3; S. H. De Boer, Canada & S. tuberosum \\
\hline UBC E155; B. Copeman, B.C., Canada & S. tuberosum & CS R6; S. H. De Boer, United States & S. tuberosum \\
\hline UBC E161; B. Copeman, B.C., Canada & S. tuberosum & C. michiganensis subsp. Michiganensis & \\
\hline 21; H. Maas Geesteranus (139), Netherlands & S. tuberosum & CM 3; A. Nelson, Canada & L. esculentum \\
\hline 441; L. Fuckikovsky (G1), Mexico & Helianthus spp. & GSPB 382; A. Mavridis, Greece & L. esculentum \\
\hline GSPB 2233; S. H. De Boer, Netherlands & S. tuberosum & GSPB 2315; Ö. Cinar, Turkey & L. esculentum \\
\hline E. chrysanthemi & & C. michiganensis subsp. nebraskensis & \\
\hline $340 ;$ E. French, Peru & S. tuberosum & GSPB 2223; M. L. Schuster, United States & Z. mays \\
\hline 571; R. Cother (DAR30511), Australia & S. tuberosum & LMG 5627; M. Schuster, United States & Z. mays \\
\hline 572; H. Maas Geesteranus ex R. Dickey (141) & Musa paradisiaca & C. michiganensis subsp. tessellarius & \\
\hline 573; H. Maas Geesteranus ex R. Dickey & & GSPB 2224; R. R. Carlson, United States & Triticum aestivum \\
\hline$(1015=$ NCPPB 1186$)$ & Parthenium argentatum & LMG 7295; A. Vidaver (78151), USA & T. aestivum \\
\hline
\end{tabular}

${ }^{a}$ Strains prefixed LMG, ATCC, BBA, and GSPB were obtained from the Culture Collection of the Laboratoriium voor Microbiologie, Rijsuniversiteit, Gent, Belgium; American Type Culture Collection, Beltsville, United States; Federal Biological Research Centre for Agriculture and Forestry, Braunschweig, Germany; or from the Göttingen Collection of Phytopathogenic Bacteria, Göttingen University, Göttingen Germany, respectively. Other strains were obtained directly from individuals as indicated. 
Selection of oligonucleotides. Taxon-specific oligonucleotides were selected from sequences of ribosomal DNA genes, the 16S23S rDNA IGS regions of pectolytic Erwinia spp. (9), and from 16S-23S rDNA sequences of $C$. michiganensis subsp. sepedonicus (24) to serve as immobilized oligonucleotides within the array (Table 2). Sequences for species-specific oligonucleotide probes for $R$. solanacearum were based on data available from GenBank (K. H. Pastrik, J. G. Elphinstone, and R. Pukall, unpublished data). Alignments were made with Pileup (Genetic Computer Group, Inc., Madison, WI) and unique polymorphisms were found manually. Selected oligonucleotides were designed for optimal and uniform hybridization kinetics using Oligo 6.3 software (1998, Molecular Biology Insights, Inc., West Cascade, CO). C6amino-terminated oligonucleotides were generated with a DNA synthesizer (Beckman Oligo 1000; Beckman Instruments, Inc., Fullerton, CA).

Preparation of the DNA array. A template of 16- to 24-mer amino-terminated oligonucleotides was prepared in the desired array format. DNA oligonucleotides were diluted from $200 \mu \mathrm{M}$ stock to $40 \mu \mathrm{M}$ in sodium bicarbonate buffer $(0.4 \mu \mathrm{M}, \mathrm{pH} 8.0)$ in a sterile 384-well microplate. Oligonucleotides were spotted with a VP 384 multi-Blot Replicator (V\&P Scientific Inc., San Diego, $\mathrm{CA}$ ) in three rows of 18 on Immunodyne ABC membranes (PALL Europe Limited, Portsmouth, England) cut into 3-by-9-cm strips. Duplicates of the same oligonucleotides were spotted on the diagonal by repeated printing. Printed membranes were air dried for $10 \mathrm{~min}$ and transferred into blocking solution $(2 \times$ standard saline citrate [SSC] [ $1 \times \mathrm{SSC}$ is $0.15 \mathrm{M} \mathrm{NaCl}$ and $0.015 \mathrm{M}$ sodium citrate], $0.5 \%$ casein [BDH Biochemical, England], and $0.05 \%$ Tween 20) and agitated for $15 \mathrm{~min}$. Blots were washed in $2 \times \mathrm{SSC}$ for $30 \mathrm{~min}$ and stored short-term in $2 \times \mathrm{SSC}$ or air dried and kept in an envelope at room temperature for long-term storage.

During the course of this study, several versions of the array were evaluated but data from only the two final arrays are presented (Fig. 1). The final version of the array included six more oligonucleotides for $E$. chrysanthemi than were used in the penultimate version.

Hybridization to DNA arrays. Before use, membranes were prehybridized for $1.5 \mathrm{~h}$ with hybridization buffer (6× SSC, $0.1 \%$ $N$-lauroylsarcosine [Sigma, L9150], 0.02\% sodium dodecyl sulfate [SDS \} [Sigma, L4509], plus 1\% casein [BDH Biochemical, Poole, England]). Dig-dUTP-labeled DNA target was denatured in boiling water for $10 \mathrm{~min}$. Overnight hybridization at $54^{\circ} \mathrm{C}$ with $6 \mathrm{ml}$ of hybridization buffer containing approximately $60 \mathrm{ng}$ of digoxigenin dUTP-labeled DNA target per membrane was followed by two washes with stringency buffer ( $4 \times$ SSC, $0.1 \%$ SDS) at hybridization temperature (22). Anti-Dig alkaline phosphatase conjugate (Roche Diagnostics GmbH, Mannheim, Germany) and the chemiluminescent substrate CDP-Star (Roche Diagnostics $\mathrm{GmbH}$ ) were used following the manufacturer's protocol. Multiple exposures of sealed membranes were done for 15 to $120 \mathrm{~min}$ on film (X-Omatic K; Kodak, Rochester, NY) on the day after adding the CDP-Star. The short exposures were enough to see all the strong reactions but, in order to find all possible cross-

TABLE 2. Name, sequence, and origin of amino-terminated 16- to 24-mer oligonucleotides used to configure DNA oligonucleotide arrays

\begin{tabular}{|c|c|c|c|}
\hline Location & Code & Sequence of oligonucleotides $\left(5^{\prime}\right.$ to $\left.3^{\prime}\right)$ & Origin of sequence ${ }^{a}$ \\
\hline A1 & Un1492 & TACGGYTACCTTGTTACGACTT & Universal 16S rDNA \\
\hline $\mathrm{A} 2$ & Un1518 & AAGGAGGTGATCCAACCGC & 16S of Erwinia spp., Escherichia coli \\
\hline A4 & Eca1127A & GCGTAATGGCGGGAAC & $16 \mathrm{~S}$ of Erwinia carotovora subsp. atroseptica \\
\hline A5 & Eca1127B & GCGTAATGGCGGGAACT & $16 \mathrm{~S}$ of E. carotovora subsp. atroseptica \\
\hline A6 & Eca1255 & GAACTCGCGAGAGCCAG & $16 \mathrm{~S}$ of $E$. carotovora subsp. atroseptica \\
\hline A7 & Eca190A & CTGTAAGTAGAGATGGGGCT & Large IGS of E. carotovora subsp. atroseptica \\
\hline A8 & Eca190B & CTGTAAGTAGAGATGGGGCTA & Large IGS of E. carotovora subsp. atroseptica \\
\hline A9 & Ech9 & АССТССТTACСАATAAAGATGT & Small IGS of E. carotovora subsp. atroseptica \\
\hline A10 & EcaCh361 & CCTTAGGGCGAGTTGTGA & Small IGS of E. carotovora subsp. atroseptica \\
\hline A11 & EcaCh244 & AGACATTATCACCGAATATCTTA & Small IGS of E. carotovora subsp. atroseptica, wasabiae \\
\hline A12 & EcaCh245 & GACATTATCACCGAATATCTT & Small IGS of E. carotovora subsp. atroseptica, wasabiae \\
\hline A13 & EcaCh370 & GAGTTGTGATGAGTCAGTGTG & Small IGS of E. carotovora subsp. atroseptica \\
\hline A14 & Ecc244A & TTAAGATATTCATGATAATGTCT & Small IGS of most $E$. carotovora subsp. carotovora \\
\hline A15 & Ecc244B & TTTAAGATATTCATGATAATGTCT & Small IGS of E. carotovora subsp. carotovora \\
\hline A16 & Ecc190 & GTAAGCAACGATGGGGTTA & Large IGS of E. carotovora subsp. carotovora, odorifera, betavasculorum \\
\hline A17 & Ecc191 & TAAGCAACGATGGGGTTA & Large IGS of E. carotovora subsp. carotovora, odorifera, betavasculorum \\
\hline B1 & EccBe9 & АССТССТTACCAAGAAGATGT & Large IGS of E. carotovora subsp. carotovora, odorifera, betavasculorum \\
\hline B2 & EccBe244 & AGACATTATCACGAATATCTTAA & Small IGS of some E. carotovora subsp. carotovora, betavasculorum \\
\hline B3 & $\mathrm{EccBe} 245$ & GACATTATCACGAATATCTTAA & Small IGS of some E. carotovora subsp. carotovora, betavasculorum \\
\hline B4 & Ebe342 & CCGTCATGCATGTTCAAGC & Small IGS of E. carotovora subsp. carotovora 441 , betavasculorum 29 \\
\hline B5 & EO1128A & GAGTTCCCGACCGAATC & $16 \mathrm{~S}$ of E. carotovora subsp. carotovora, odorifera, betavasculorum \\
\hline B6 & EO128B & TGAGTTCCCGACCGAATC & $16 \mathrm{~S}$ of E. carotovora subsp. carotovora, odorifera, betavasculorum \\
\hline B7 & EO1255 & CTTGCTCTCGCGAGGTC & $\begin{array}{l}\text { 16S of } E \text {. carotovora subsp. carotovora, odorifera, betavasculorum, wasabiae, E. } \\
\text { chrysanthemi }\end{array}$ \\
\hline B8 & Ech171 & GACGGTGGGTGAAAGGC & Small IGS of E. chrysanthemi \\
\hline B9 & Ech194 & AACGCTAACCTAAAACTGATT & Small IGS of E. chrysanthemi \\
\hline B 10 & Ech 165 & ATGACTGACGGTGGGTGAA & Small IGS of E. chrysanthemi \\
\hline B11 & Ech190 & GGTCAACGCTAACCTAAAACT & Small IGS of E. chrysanthemi \\
\hline B14 & EchUp333 & TCCCGAGACACTTTCGG & Small IGS of E. chrysanthemi \\
\hline B15 & Ech333 & CCGAAAGTGTCTCGGGA & Small IGS of E. chrysanthemi \\
\hline $\mathrm{C} 2$ & RaSo41 & CGTGCATTCTAGTTAGGCG & IGS of Ralstonia solanacearum \\
\hline $\mathrm{C} 3$ & RalSo180 & ACGGTGGAAGTCTCTGCC & IGS of $R$. solanacearum \\
\hline $\mathrm{C} 4$ & RaSo299 & CGCAAGCATCGAGTTTTC & IGS of $R$. solanacearum \\
\hline $\mathrm{C} 5$ & RaSo333 & ATTGCCAAGACGAGTAATAAC & IGS of $R$. solanacearum \\
\hline C6 & RaSo405 & ATGAGATGCTCGCAACAAC & IGS of $R$. solanacearum \\
\hline $\mathrm{C} 7$ & RaSo460 & GAGTGATCGAAAGACCGCT & IGS of $R$. solanacearum \\
\hline $\mathrm{C} 8$ & Cms181 & GGGTGGGAAAATGGTCTG & IGS of Clavibacter michiganensis subsp. sepedonicus \\
\hline C9 & Cms182 & GGTGGGAAAATGGTCTG & IGS of C. michiganensis subsp. sepedonicus \\
\hline $\mathrm{C} 10$ & $\mathrm{Cms} 250 \mathrm{~A}$ & ACCAGACACACCAAAAGG & IGS of $C$. michiganensis subsp. sepedonicus \\
\hline $\mathrm{C} 11$ & Cms250B & AACCAGACACACCAAAAGG & IGS of $C$. michiganensis subsp. sepedonicus \\
\hline $\mathrm{C} 12$ & $\mathrm{Cms} 250 \mathrm{C}$ & AAACCAGACACACCAAAAGG & IGS of $C$. michiganensis subsp. sepedonicus \\
\hline
\end{tabular}

a IGS = intergenic spacer region. 


\begin{tabular}{|c|c|c|c|c|c|c|c|c|c|c|c|c|c|c|c|c|c|c|c|c|c|c|c|c|c|c|c|c|c|c|c|c|}
\hline & & & & & & & & & & & & & blig & one & ucle & otid & de lo & ocat & ation & $\mathrm{n}$ an & idc c & cod & & & & & & & & & & \\
\hline & & & & & $\alpha$ & & 25 & & & $\frac{m}{<}$ & $\frac{5}{<}$ & $\frac{\infty}{4}$ & $\frac{9}{4}$ & $\hat{s}$ & & & & & & & & & & $\frac{\nabla}{9}$ & & & & & 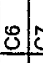 & & & \\
\hline PCR template for probe & & 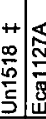 & & 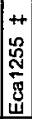 & & & 它 & & 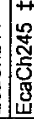 & 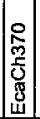 & 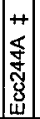 & & 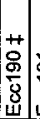 & & & & & & 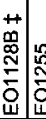 & & 㕝 & & & 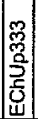 & 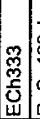 & $\mid$ & & & & & & 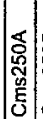 \\
\hline E. c. subsp. atroseptica 1401 & 而 & $\overrightarrow{0}$ & 遌 & $\square$ & 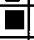 & 㟧 & 口 & {[} & te & a & & & & & & & & & & & & 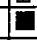 & & & & & & & & & & \\
\hline E. c. subsp. atroseptica 1700 & 口 & Q두 & {[} & $\square$ & Q & 口 & $\square[$ & 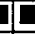 & {[} & D & & & & & & & & & & E & $=$ & - & - & - & - & & & & & & & \\
\hline E. c. subsp. atroseptica 196 & $\square$ & $\square$ & [0 & $\square$ & E & $\square$ & DI & {[} & $E$ & $\mathbf{D}$ & & & & E & 氜 & & $\cdot$ & $\cdot$ & & 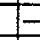 & $E$ & E & - & - & - & & & & & & & \\
\hline E. c. subsp. atroseptica $31^{*}$ & $\square$ & $\square[$ & $E$ & $\square$ & 可 & $\square$ & $\square[$ & E & 10 & 且 & & & & & & & & & & & & - & & & & & & & & & & \\
\hline E. c. subsp. atroseptica 558 & 回 & DE & D & $\mathbf{a}$ & a & & Q & ] & ] & a & & & & & & & & & & E & - & - & - & - & - & & & & & & & \\
\hline E. c. subsp. atroseptica 9201 & 口 & 口[ & D & $\square$ & 口 & D & प्र & ] [ & t & 四 & & & & & & & & & & - & -1 & $1-$ & - & -1 & E & & & & & & & \\
\hline E. c. subsp. atroseptica 9204 & $\mathbf{D}$ & 可 & $E$ & $\square$ & 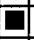 & Z & 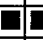 & 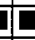 & E & a & & & & & & & & & & - & - & - & - & - & -1 & & & & & & & \\
\hline E. c. subsp. atroseptica 9205 & 国 & $\square[\bar{G}$ & {[} & $\square$ & $\square$ & 品 & DE & a & I & a & & & & & & & & & & - & -1- & - & - & $1-$ & - & & & & & & & \\
\hline E. c. subsp. atroseptica 2231 & $\square$ & Q & {[} & $\square$ & E & $\square$ & 7 & E & IL & $\mathbf{D}$ & & & & & & & & & & & & 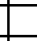 & t & & & & & & & & & \\
\hline E. c. subsp. wasabiae 91 & 田 & $\square$ & & & & & & E & I & & & & & & & & & & & & & & & & & & & & & & & \\
\hline E. c. subsp. wasabiae 92 & 回 & 回 & & & & & & E & 1 & & & & & & & & & & E & 可 & & 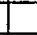 & & & 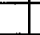 & & & & & & & \\
\hline E. c. subsp. wasabiae 93 & Di & [a & & & & & 回 & E & 口 & & & & & & & & & & E & a & & & & & 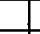 & & & & & & & \\
\hline E. c. subsp. wasabiae 94 & 可 & ( & & & & & & E & 國 & & & & & & & & & & E & 可 & & . & 1 & 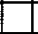 & . & & & & & & & \\
\hline E. c. subsp. atroseptica E1 & al & $\square$ & & & & & & & & & 可 & 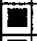 & $\square$ & $\square$ & 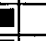 & & & $\square$ & $\square$ & - & - & E & - & $=$ & - & & & & & & & \\
\hline E. c. subsp. carotovora 21 & Q1 & 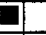 & & & & & & & & & 回 & Q & DI & DI & ] & & & $\square[$ & $\square[$ & 可 & $1-$ & - & - & -1 & -1 & & & & & & & \\
\hline E. c. subsp. carotovora E155 & Q & 回 & & & & & & & & & 回 & 可 & Q1 & 国 & ] & & & E & ED & ] & - & - & - & - & -1 & & & & & & & \\
\hline E. c. subsp. carotovora E161 & DI & $\square$ & & & & & & & & & D & $\mathbf{a}$ & DI & DI & ] & & & $\mathbf{\square} \mathbf{2}$ & $\mathbf{D}[$ & ]- & $-1-$ & - & - & E & -1 & & & & & & & \\
\hline E. c. subsp. carotovora 2233 & DI & $\square$ & & & & & & & & & & 回 & $\square[$ & 可 & ] & & & 可 & DE & & & - & 1 & 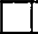 & $\square$ & & & & & & & \\
\hline E. c. subsp. odorifera $1645-1$ & 四 & Q & & & & & & & & & & & $\nabla[$ & 可 & 可 & & & 可 & D & & & - & 1 & & & & & & & & & \\
\hline E. c. subsp. odorifera 1878 & 畐 & 田 & & & & & & & & & & & $\square[$ & 可 & ] & & & 回 & Q & 可 & & 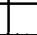 & 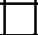 & & 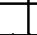 & & & & & & & \\
\hline E. c. subsp. odorifera 1880 & Di & Q & & & & & & & & & 田 & D & DI & 可 & & & & QI & $\square[$ & ] & & 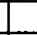 & L & 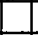 & 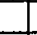 & & & & & & & \\
\hline E. c. subsp. odorifera 1893 & D & $\square$ & & & & & & & & & & 田 & $\square$ & Q & & & & Q & 口[ & ] & & 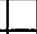 & D & & & & & & & & & \\
\hline E. c. subsp. betavasculorum 29 & $\square$ & $\square$ & & & & & & & & & & & DI & 可 & $\bar{\sigma} \mathbf{E}$ & $\square$ & 回 & 口[ & QE. & ]- & $-1-$ & - & - & - & - & & & & & & & \\
\hline E.c. subsp. carotovora 441 & DI & $\square$ & & & & & & & & & & & Q & DI & 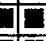 & Q & $\bar{I}$ & $\square$ & E. & 可 & - & - & - & - & - & & & & & & & \\
\hline E. chrysanthemi 413 & Di & [ & & & & & & & & & $\cdot 1$ & 可 & 日1 & D[ & {$[\bar{a}$} & D & & 可 & 口E & - & - & - & - & - & - & & & & & & & \\
\hline E. chrysanthemi 1406 & Q1 & a & & & & & & & & & & & QD & 可 & $\mathbf{\square}$ & 口 & & 可 & $\mathbf{\square}$ & E & - & - & - & - & -1 & & & & & & & \\
\hline E. herbicola 2565 & 口i & ] & & & & & & & & & & & & & & & & D] & 国 & ]- & - & - & -1 & $=$ & -1 & & & & & & & \\
\hline E. herbicola 450 & $\mathbf{\square}$ & $\square$ & & & & & & & & & & & & & & & & 可 & $\mathbf{\square}$ & - & - & - & - & -1 & -1 & & & & & & & \\
\hline E. stewartii 2626 & 回 & 国 & & & & & & & & & & & & & & & & DE & $E[$ & 可— & $=$ & - & - & - & -1 & & & & & & & \\
\hline E. chrysanthemi 572 & $\square$ & $\square$ & & & & & & & & & & & & & & & & & & & & 可 & 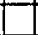 & 可 & & & & & & & & \\
\hline E. chrysanthemi $340^{*}$ & D & 五 & & & & & & & & & & & & & & & & & E. & 司 & 可 & $\mathbf{\square}$ & $\square$ & $\mathbf{D}_{1}$ & 可 & & & & & & & \\
\hline E. chrysanthemi 571 & $\square$ & a & & & & & & & & & & & & & & & & & E & ] - & $\square$ & $\square$ & $\square$ & $\mathbf{D}_{\mathbf{b}}$ & 可 & & & & & & & \\
\hline E. chrysanthemi 573 & $\mathbf{\square}$ & a & & & & & & & & & & & & & & & & & & 四 & a & $\square$ & a & & 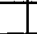 & & & & & & & \\
\hline E. chrysanthemi 580 & $\mathbf{0}$ & 可 & & & & & & & & & & & & & & & & & & 므 & {[} & 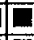 & a & & & & & & & & & \\
\hline E. chrysanthemi 582 & D & ] & & & & & & & & & & & & & & & & & 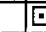 & 可 [ & ] & $\bar{a}$ & a & & . & & & & & & & \\
\hline R. solanacearum 1410 & $\square$ & 可 & & & & & & & & & & & & & & & & & & 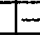 & - & - & - & - & -1 & $\square[$ & & & & & & \\
\hline R. solanacearum 1424 & $\mathbf{\square}$ & & & & & & & & & & & & & & & & & & & & - & - & - & E & -1 & $\square$ & 可 & & & & & \\
\hline R. solanacearum 2300 & 口 & & & & & & & & & & & & & & & & & & & & & L & 1 & & 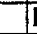 & & $\square$ & & & & & \\
\hline R. solanacearum 2305 & 1 & & & & & & & & & & & & & & & & & & & & & 1 & & & & $\mathbf{\square}$ & {$[\mathbf{E}$} & & & & & \\
\hline R. solanacearum 2695 & ( & & & & & & & & & & & & & & & & & & & & & - & - & -1 & -1 & 更 & & 可 & & & & \\
\hline R. solanacearum 2763 & a & & & & & & & & & & & & & & $\cdot$ & & & & & & & D & 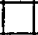 & 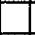 & 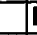 & $\square$ & & (a) & & & & \\
\hline R. solanacearum 2791 & $\square$ & & & & & & & & & & & & & & & & & & & & E & - & - & 二 & -1 & $\underline{\square}$ & & 1 & 国 & & & \\
\hline R. solanacearum 551 & $\square$ & & & & & & & & & & & & & & & & & & & & & - & - & 4 & -1 & 而 & & in & (1] & ] & & \\
\hline P. fluorescens 1714 & t & & & & & & & & & & & & & & & & & & & 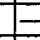 & - & - & - & Z & - & & & & & & & \\
\hline C. m. subsp. tessellarius & & & & & & & & & & & & & & & & & & & & & & & & & & & & & & & & \\
\hline C. $m$. subsp. tessellarius 2224 & 口 & & & & & & & & & & & & & & & & & & & & & & & & & & & & & & & \\
\hline C. m. subsp. nebraskensis 5627 & $\square$ & & & & & & & & & & & & & & & & & & & & & & & & & & & & & & & \\
\hline C. m. subsp. nebraskensis 2223 & $\square$ & & & & & & & & & & & & & & & & & & & & & $\mathbf{E}$ & & & & & & & & & & \\
\hline C. m. subsp. insidiosus 2225 & 表 & & & & & & & 可 & & $\mathbf{a}$ & & & & & & & & & & & & & & & & & & & & & & \\
\hline C. m. subsp. michiganensis 382 & $\mathbf{\square}$ & & & & & & & & & & & & & & & & & & & & & & & & & & & & & & & \\
\hline C. m. subsp. michiganensis 3 . & [ & & & & & & & & & & & Q & & & & & & & & & & & & & & & & & & & & \\
\hline C. m. subsp. michiganensis 2315 & 口 & & & & & & & & & & & & & & & & & & & & & & & & & & & & & & & \\
\hline C. $m$. subsp. sepedonicus $\mathbf{R}^{*}$ & 回 & & & & & & & & & & & & & & & & & & & & & $\square$ & & & & & & & & & a & - \\
\hline C. m. subsp. sepedonicus R6 & 口 & & & & & & & & & & & & & & & & & & & & & . & & - & 7 & & & & & & 回 & 可 \\
\hline C. m. subsp. sepedonicus 12 & & & & & & & & & & & & & & & & & & & & & E & - & & - & 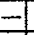 & & & & & & 12 & a \\
\hline C. m. subsp. sepedonicus $3 \mathrm{M}$ & 口 & & & & & & & & & & & & & & & & & & & & - & - & $1-$ & -1 & -1 & & & & & & 10 & - \\
\hline C. m. subsp. sepedonicus $150 / 100$ & $\square$ & & & & & & & & & & & & & & & & & & & & - & - & $1-$ & -1 & - & & & & & & 回 & 可 \\
\hline C. m. subsp. sepedonicus $R 10$ & $\mathbf{a}$ & & & & & & & & & & & & & & & & & & & $E$ & - & - & - & - & \pm & & & & & & 回 & \\
\hline Escherichia coli 0157:H7 & a & & & & & & & & & & & & & & & & & & & E & - & - & - & - & - & & & & & & & \\
\hline Listeria monocytogenes & 田 & & & & & & & & & & & & & & & & & & & & - & - & & 二 & - & & & & & & & \\
\hline tissue $\mathrm{ct}$ & L & & & & & & & & & & & & & & & & & & & & & & & & & & & & & & & \\
\hline potato infected with E. c. atros. $\dagger$ & DI & & & & & & GE & & & D & & & & & & & & ] & 口[ & & & & & & & & & & & & & \\
\hline E. c. atros. $31+$ E. chrys. & (1) & $\overline{0}$ & & & & & TE & & & 口 & & & & & & & & & & & $\overline{\mathbf{Z}}$ & I & $\square$ & -1 & & & & & & & & \\
\hline
\end{tabular}

Fig. 1. Hybridization of digoxigenin-labeled polymerase chain reaction amplicons from selected bacterial species and samples to oligonucleotides immobilized on nylon membrane in an array. Hybridization signal strength of each amplicon to an oligonucleotide in the array is expressed in 16-bit grayscale values indicated by the following symbols: $\square=\langle 500 ; \cdot=500$ to 1,000 ; $\square=1,000$ to 10,000 ; $\square=10,000$ to 20,000 ; $\mathbf{\square}=20$,000 to 30,000 ; $\mathbf{\square}=30,000$ to 65 , 000 ; and $-=$ not tested because these six oligonucleotides were present only on a final membrane version. The location of oligonucleotides in the array given at the top of the figure correspond to their location on the membranes in Figures 2 and 3; oligonucleotides are described in Table 2. $*=$ membranes shown in Figure 2. $\dagger=$ membranes shown in Figure 3 . 
reacting oligonucleotides, the films that gave the maximum number of dots before the background became too dark (i.e., $45 \mathrm{~min}$ or more of exposure) were selected for scanning and analyses.

Chemiluminogrammes were scanned at 16-bit gray using Fotolook Ps 2.08 software on an ARCUS II scanner (AGFA, Taiwan, ROC). Gray values for each spot were evaluated using GenePix Pro 3.0.6 (AXON Instruments, Inc., CA).

Membrane arrays were reused after stripping off hybridized DNA with $0.2 \mathrm{M} \mathrm{NaOH}$ at $65^{\circ} \mathrm{C}$ in a hybridization oven for $30 \mathrm{~min}$, rinsing with $2 \times \mathrm{SSC}$, and washing with $0.5 \times \mathrm{SSC}$ for $20 \mathrm{~min}$ at $65^{\circ} \mathrm{C}$. Stripped membranes were transferred to $2 \times \mathrm{SSC}$ and agitated for $30 \mathrm{~min}$, and were ready for use or stored as described previously.

Mixed bacteria and potato samples. To test the discriminatory potential of the oligonucleotide array, suspended bacterial cells of E. carotovora subsp. atroseptica (strain 31 ) and E. chrysanthemi (strain 340) were mixed 1:1 prior to DNA extraction. Genomic DNA mixture combinations of $C$. michiganensis subsp. sepedonicus (strains CS 12 and CS 150/100), E. carotovora subsp. atroseptica (strains 558 and 196), E. carotovora subsp. carotovora (strains 21 and 441), E. chrysanthemi (strains 573 and 340), and $R$. solanacearum (strains 511 and 2791) were mixed 1:1 (10 to $15 \mathrm{ng} / \mu \mathrm{l}$ for each strain) prior to PCR amplification.

DNA extracted from micropropagated noninfected potato plantlets was used as a control for hybridizations to the array. In addition, potato tubers were inoculated with E. carotovora subsp. atroseptica at approximately $1 \times 10^{6} \mathrm{CFU} / \mathrm{ml}$ by vacuum infiltration as described (27). DNA from inoculated and uninoculated tubers were amplified and labeled with digoxigenin-11-dUTP by PCR using primer set 1114f/L1r. Labeled DNA was denatured in boiling water for $10 \mathrm{~min}$ before hybridizing to arrays using the same procedure as for DNA from pure bacterial colonies.

\section{RESULTS}

Development of the oligonucleotide array. Forty specific oligonucleotides were designed for the main potato bacterial

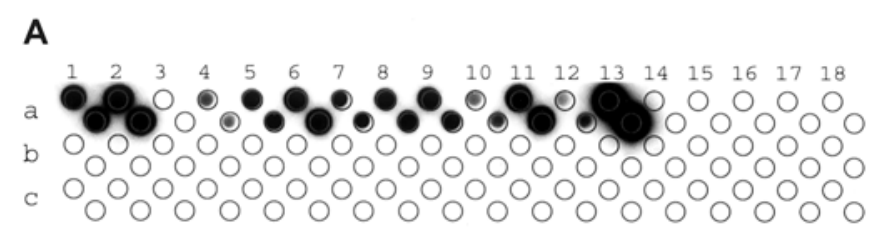

B

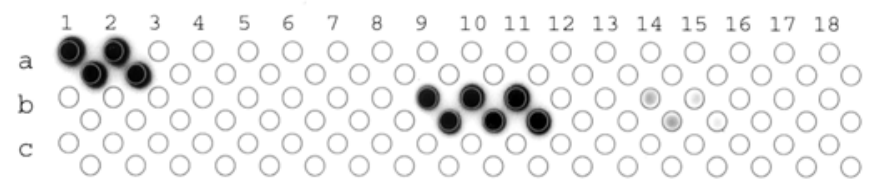

C

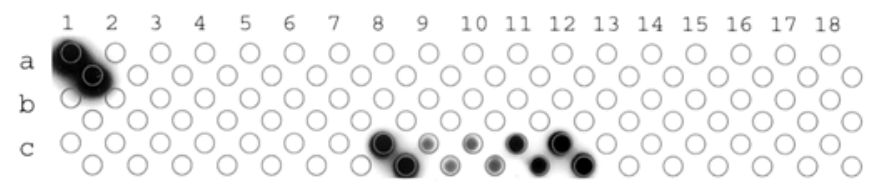

Fig. 2. Hybridization pattern of digoxigenin-labeled polymerase chain reaction amplicons from $\mathbf{A}$, Erwinia carotovora subsp. atroseptica strain 31; B, E. chrysanthemi strain 340; C, and Clavibacter michiganensis subsp. sepedonicus strain R3 on the oligonucleotide array. Oligonucleotides were spotted, with duplicates on the diagonal, in three rows (a, b, and c) of 18 spots. After hybridization, detection, and film exposure, a template was placed over the membrane to identify spot locations. Positive hybridization signals are visualized as dark spots within template circles and were analyzed on the basis of gray scale values summarized in Figure 1. pathogens using the $3^{\prime}$ end of the $16 \mathrm{~S}$ gene or the IGS regions that are bracketed by the $16 \mathrm{~S}$ and $23 \mathrm{~S}$ ribosomal genes. The oligonucleotides were designed with comparable melting temperatures. Multiple oligonucleotides were selected on the basis of specificity for each bacterial species (Table 2). For example, for E. carotovora subsp. atroseptica, three oligonucleotides (Eca1127A, Eca1127B, and Eca1255) were designed from the $16 \mathrm{~S}$ sequence, two (Eca190A and Eca190B) were from the IGS region of the larger ribosomal operon, and three (Ech9, EcaCh361, and EcaCh370) from the IGS region of the smaller ribosomal operon. Another four oligonucleotides (EcaCh244, EcaCh245, Ecc244A, and Ecc244B) were designed from common IGS sequences within E. carotovora subsp. atroseptica and wasabiae or subsp. carotovora, respectively. Seventeen oligonucleotides were designed with specificity to E. carotovora subspecies and E. chrysanthemi, six with specificity to $R$. solanacearum, and five with specificity to $C$. michiganensis subsp. sepedonicus (Table 2). Prior to synthesis and incorporation into the array, oligonucleotide sequences were screened by Blastn searches for homology to bacterial sequences in GenBank.

Two oligonucleotides (Un1492 and Un1518), designed as universal targets, served as the positive controls. Several locations on the array (A3, B12-13, B16-18, C1, and C13-18) not spotted with oligonucleotides served as negative controls. Six additional oligonucleotides (Ech171, Ech194, Ech165, Ech190, EchUp333, and Ech333) were designed for the final version of the array and are shown as missing values for the first data set (Fig. 1).

Evaluation of the specificity of the oligonucleotide array. A fragment of the ribosomal gene cluster was amplified by PCR, using conserved primers, from each bacterial strain used in this study (Table 1). Digoxigenin-labeled PCR amplicons generated from pure cultures of bacteria generally hybridized to the array according to the expected patterns (Fig. 1). Patterns were highly reproducible in the two to three hybridizations conducted with each of the bacterial templates.

Hybridization signal strength, expressed in gray values, ranged from 0 to 65,000 among the oligonucleotide spots on the arrays. Gray values of $>500$ were arbitrarily considered as positive hybridization signals while lower values, not visible without computer enhancement, were considered to be background signals. Exposures were selected for analysis that gave signal-to-noise ratios, based on positive and negative controls, $>4,400: 1$. Mean gray value for spots regarded as negative was 10.7 , whereas the mean of positive signals was 39,460 .

Labeled amplicons generated from nine strains of $E$. carotovora subsp. atroseptica hybridized to the 10 oligonucleotides designed for the subspecies (Fig. 1). Only two strains (Eca1401 and Eca196) hybridized to oligonucleotides designed for other erwinias (Ech165, EccBe9, Ebe342, and EO128A) but at least in one case (Eca1401), the cross-hybridization could not be duplicated (the membrane that gave a cross reaction is shown in Fig. 1). This was an exception in duplication of results because hybridization with amplicons from the same strain normally showed the same hybridization pattern. The variation in intensity of hybridization to different oligonucleotides is exemplified with strain 31 (Fig. 2A); the amplicon hybridized strongly to oligonucleotide EcaCh370 (location A13) but more weakly with oligonucleotide Eca1127A (location A4). The amplicon generated from strain 31 did not cross-hybridize with heterologous oligonucleotides but did hybridize with the positive control oligonucleotides (Fig. 2, location A1-2). Strain E1, received as E. carotovora subsp. atroseptica, was an exception in that it did not hybridize to the homologous oligonucleotides but rather presented a hybridization pattern that was the same as other strains of E. carotovora. subsp. carotovora. This confirms that this strain is likely misnamed, as discussed previously (9). Oligonucleotides EcaCh244 and EcaCh245 were selected as common sequences of E. carotovora subsp. atroseptica and wasabiae, and the unique hybridization of the E. caroto- 
vora subsp. wasabiae to these oligonucleotides was consistent with the results expected from the sequences (Fig. 1). Strains of $E$. carotovora subsp. betavasculorum, carotovora, and odorifera similarly presented hybridization patterns consistent with the oligonucleotide and rDNA sequence comparisons (Table 2; Fig. 1).

The reaction of E. chrysanthemi 340 was typical for the species which hybridized strongly to three oligonucleotides (Ech194, Ech165, and Ech190) (Figs. 1 and 2B locations B9-11, respectively). Weaker hybridizations were noted with oligonucleotides EO1255 (Fig. 2B, not visible at location B7) and EChUp333 and Ech333 (Fig. 2B, location B14-15). Hybridization patterns for $E$. chrysanthemi strains 1406 and 413 were consistent with sequencing data which showed that these strains are likely misnamed (9). These strains were previously shown to be similar to E. carotovora subsp. carotovora and their hybridization patterns were consistent with this observation (Fig. 1). Strains of E. herbicola and E. stewartii hybridized with two to three oligonucleotides designed for specificity to E. carotovora subspecies but not with eight other oligonucleotides that characterized many $E$. carotovora strains.

Amplicons generated from all six strains of $C$. michiganensis subsp. sepedonicus tested hybridized to the five homologous oligonucleotides (Figs. 1 and 2C locations C8-12). Other subspecies of C. michiganensis, including michiganensis, insidiosus, nebraskensis, and tessellarius, did not hybridize to oligonucleotides designed from the IGS region of $C$. michiganensis subsp. sepedonicus (Fig. 1). Only six out of a few hundred heterologous spots probed with Clavibacter DNA produced a signal. These spurious hybridizations did not interfere with bacterial identifications. All eight strains of $R$. solanacearum hybridized to oligonucleotides RaSo460 and RaSo41, but the strains hybridized differentially to the other four R. solanacearum-specific oligonucleotides (Fig. 1). $R$. solanacearum amplicons did not hybridize to oligonucleotides designed from sequences of heterologous species except for a single weak heterologous hybridization signal among all the heterologous spots probed with $R$. solanacearum DNA (Fig. 1).

An amplicon from Escherichia coli O157:H7, Listeria monocytogenes, and Pseudomonas fluorescens only hybridized with one or both the universal oligonucleotide controls, as expected (Fig. 1).

Discriminatory potential of the oligonucleotide array. Several mixtures of bacterial strains were amplified by PCR with the conserved primers and hybridized to the oligonucleotide array. The mixture of amplicons did not interfere with one another and the expected pattern for each species was obtained. An array probed with amplicons from a mixture of Erwinia carotovora subsp. atroseptica strain 31 and E. chrysanthemi strain 340 is presented in Figure 3A and summarized at the bottom of Figure 1. The hybridization pattern is consistent with that expected for the two Erwinia spp.

Bacteria also could be identified directly in potato tissue using the oligonucleotide array. PCR amplicons generated from potato tubers inoculated with E. carotovora subsp. atroseptica strain 31 produced the same hybridization pattern on the array as pure cultures (Figs. 3B and 1 bottom), again confirming the reproducibility of hybridization pattern. The hybridization to the three oligonucleotides (EO1128A, EO1128B, and EO1255) characteristic of E. herbiolca (Pantoea agglomerans) and E. stewartii ( $P$. stewartii subsp. stewartii) suggests that there were nonpectolytic enterobacteria among the potato microflora. Bacteria-free potato tissue from microplantlets did not hybridize with any of the oligonucleotides designed for the pathogenic bacteria (Figs. 3C and 1 bottom).

\section{DISCUSSION}

Traditional methods for identification of bacteria require the isolation of pure cultures of the organism to be identified. In- creasingly, however, molecular methods can be used to identify specific target species within mixtures of microorganisms without prior isolation of pure cultures $(25,26)$. DNA array technology is one such method that has potential for rapid and cost-effective identification of pathogenic bacteria in the complex microbial communities which are normally associated with plants $(1,21$, 36,38).

In our study, we evaluated the potential usefulness of DNA array technology for identifying bacteria involved in potato pathogenesis and differentiating them from closely related strains. We targeted the ribosomal operon as a source of species- and subspecies-specific oligonucleotides because not only are sequences of this gene region available for numerous bacteria, but they also have been shown to be useful for detection and identification of various plant pathogenic bacteria $(16,17,33)$.

By targeting the ribosomal gene, we were able to use conserved primers for amplifying template DNA across a wide range of bacterial species. The rDNA sequence homology is high among eubacteria; therefore, the 16S and IGS chromosomal regions of any of the plant-pathogenic bacteria could be amplified with a single primer pair (19). Hence, for any field sample, amplicons can be generated without the need for preliminary identification of a target species. The pathogenic species within the sample would be identified by the hybridization pattern on the array. The sensitivity threshold for detecting pathogenic strains among a large number of nonpathogenic bacteria needs to be determined. It is interesting to point out again that nonpathogenic species were detected in a potato sample inoculated with E. carotovora subsp. atroseptica (Fig. 1).

With the hybridization stringency used in our study, the selected oligonucleotides generally displayed the desired specificity. The oligonucleotides were selected to have at least two base differences from closely related species or subspecies. For example, the oligonucleotides from the IGS region of $C$. michiganensis subsp. sepedonicus (Cms181-2; Table 2) differed from the other
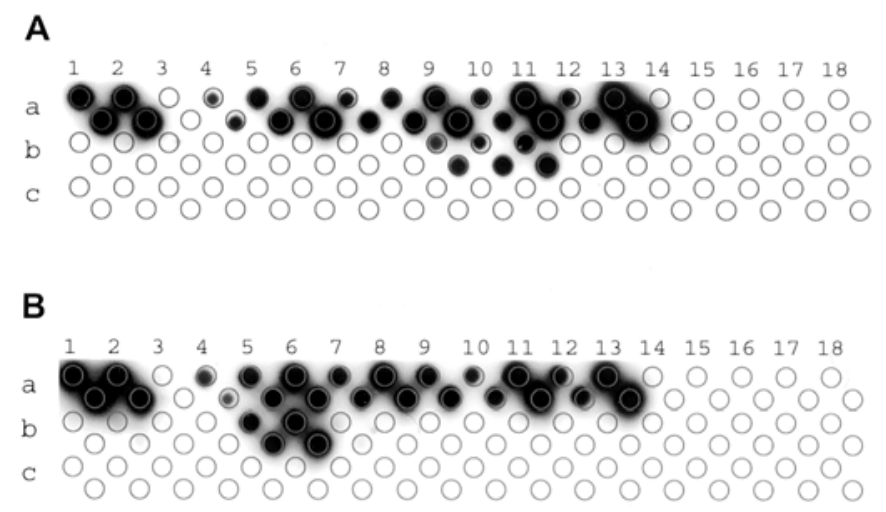

\section{C}

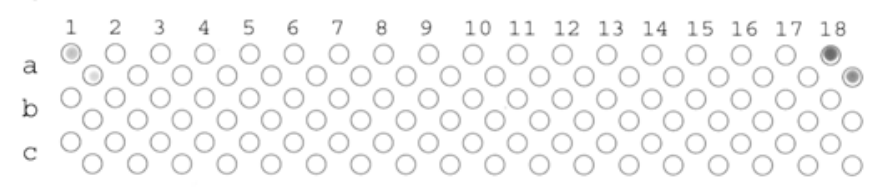

Fig. 3. Hybridization pattern of digoxigenin-labeled polymerase chain reaction amplicons obtained from $\mathbf{A}$, a mixture of Erwinia carotovora subsp. atroseptica strain 31 and E. chrysanthemi strain 340 cells; B, DNA from $E$. carotovora subsp. atroseptica-inoculated potato tuber; and C, DNA from potato tissue culture. Oligonucleotides were spotted, with duplicates on the diagonal, in three rows ( $a, b$, and c) of 18 spots. After hybridization, detection, and film exposure, a template was placed over the membrane to identify spot locations. Positive hybridization signals are visualized as dark spots within template circles and were analyzed on the basis of gray scale values summarized in Figure 1. 
subspecies $C$. michiganensis subsp. michiganensis and insidiosus by this minimum of two nucleotides (24). Gray values from hybridization of strains of $C$. michiganensis subsp. sepedonicus to oligonucleotide Cms181 varied between 31,000 and 59,000, whereas they varied between 0 and 94 for $C$. michiganensis subsp. michiganensis and insidiosus (Fig. 1). Unlike primers designed for PCR, discriminatory specificity is greatest when mismatches are in the center of the immobilized oligonucleotide (18); therefore, we had to modify the PCR primer Sp1f (24) into Cms181 and Cms182 for hybridization purposes.

The diversity among the pectolytic Erwinia spp. provided special challenges for developing discriminatory oligonucleotides. Whereas E. carotovora subsp. atroseptica forms a distinct and uniform taxonomic cluster closely related to subsp. wasabiae strains (9), it is distinguishable from other E. carotovora strains. Differentiation of E. carotovora subsp. carotovora from subsp. odorifera and betavasculorum would be challenging using the oligonucleotides selected for our array (Fig. 1). Nevertheless, some apparently misidentified strains, such as strains E1, 413, and 1406 , could be placed in the correct taxa on the basis of their hybridization patterns.

The E. chrysanthemi strains that cause a serious blackleg-like disease on potato are difficult to differentiate from strains of this species that are pathogenic on other plants $(2,15)$. Despite the heterogeneity within the species, as demonstrated by the work of Dickey (5) and Dickey et al. (6) with phenotypic and serological characteristics, a wide variety of strains in our study showed a distinctive E. chrysanthemi hybridization pattern on the array. It is perhaps not surprising that the banana strain 572 did not hybridize with most $E$. chrysanthemi-specific oligonucleotides.

The ease with which species- and subspecies-specific oligonucleotides can be selected depends to a considerable extent on the genetic uniformity of the taxon. $C$. michiganensis subsp. sepedonicus is phenotypically very uniform and this was reflected in the consistent hybridization pattern with isolates from regions as diverse as British Columbia, New Brunswick, and North Dakota (Table 1; Fig. 1). In contrast, strains of $R$. solanacearum showed greater diversity in hybridization pattern. Only one strain clearly hybridized with all six oligonucleotides selected, whereas two out of eight strains hybridized to only two oligonucleotides. This is not surprising given the diversity among race 3 strains found on potato in Europe alone (35) and the fact that our study included race 1 and 3 strains from widely separated geographic regions. Testing and sequencing of additional strains will no doubt more clearly delineate signature oligonucleotides of greatest value in an array.

Hybridization of labeled amplicons to our oligonucleotide array resulted in distinct patterns that were sufficient for making reliable presumptive identification of a species or subspecies and discriminated target species from closely related bacteria. However, because a difference in oligonucleotide sequences as small as two bases was sufficient to prevent hybridization, multiple oligonucleotides were required to consistently detect all strains within a species. Variation among strains can result in the failure of hybridization to any one oligonucleotide that is diagnostic for a species or subspecies. Although some bacterial taxons, such as $C$. michiganensis subsp. sepedonicus, as already noted, are remarkably uniform, variation in others, such as E. carotovora subsp. carotovora and $R$. solanacearum, is much greater. We estimate that four to five specific oligonucleotides per bacterial taxon might be required in an array to provide an adequate fingerprint to identify an unknown isolate.

Spurious hybridization of amplicons to oligonucleotides of unrelated taxons can be expected at low frequency due to coincidental similarities in base sequences of unrelated genes amplified at low level, because of similar gene sequences in taxonomically unrelated bacteria or through PCR artifacts. Such spurious hybridizations were minimized by using oligonucleotides of 16 to
24 bases in length. Nevertheless, a small number of bacteria hybridized with heterologous oligonucleotides. These hybridizations were generally weak and in no instance interfered with recognition of taxon-specific hybridization patterns.

We evaluated the ability of the oligonucleotide array to identify bacteria in microbial mixtures and in field samples only in a very preliminary way. The results, however, were encouraging, in that the anticipated hybridization patterns were obtained (Fig. 3). The hybridization pattern of our field sample (Fig. 3B) suggested the presence of a nonpectolytic enterobacteria, probably E. herbicola, a common plant epiphyte. Its presence did not interfere with the identification of E. carotovora subsp. atroseptica in the sample; instead, it illustrated the potential power of arrays for analyzing environmental samples.

Oligonucleotide array technology should be useful in the rapid identification of bacterial pathogens of potato. The utility of such an array would be further enhanced by the addition of oligonucleotides specific for other potato pathogens, including fungi, nematodes, and perhaps even viruses. Several PCR reactions may be required with conserved or degenerate primers, or with a reverse transcription step for RNA viruses, to produce labeled amplicons for the various pathogen groups. Production of the array on alternate platforms such as glass slides or electronic chips would enhance the efficacy and ease of use. In the future, a set of pathogen-specific oligonucleotides could be combined with other oligonucleotides specific to cultivars and genetic transformations to provide a single multipurpose array for the potato industry.

\section{ACKNOWLEDGMENTS}

We thank S. Farah and P. Delaquis for technical assistance with the processing of human pathogens; L. Ward, Canadian Food Inspection Agency, Centre for Animal and Plant Health, Charlottetown, PEI, Canada, for providing potato samples and for reviewing the manuscript; M. J. Coté and P. Delaquis for critical review of the manuscript; F. Niepold, BBA Braunschweig, Germany, and A. Mavridis, Curator of GSPB Collection, Institute of Plant Pathology, Göttingen University, Germany, for providing bacterial cultures; the personnel of the graphic department at Agriculture and Agri-Food Canada, Eastern Cereal and Oilseed Research Centre, Ottawa, Ontario, K1A OC6 for valuable assistance; and the NSERC Canadian Government Laboratory Visiting Fellowship and the Canadian Biotechnology Strategy program.

\section{LITERATURE CITED}

1. Anthony, R. M., Brown, T. J., and French, G. L. 2000. Rapid diagnosis of bacteria by universal amplification of $23 \mathrm{~S}$ ribosomal DNA followed by hybridization to an oligonucleotide array. J. Clin. Microbiol. 38:781788 .

2. Boccara, M., Vedel, R., Lalo, D., Lebrun, M. H., and Lafay, J. F. 1991. Genetic diversity and host range in strains of Erwinia chrysanthemi. Mol. Plant-Microbe Interact. 4:293-299.

3. De Boer, S. H. 1994. The role of plant pathology research in the Canadian potato industry. Can. J. Plant Pathol. 16:150-155.

4. De Boer, S. H., and Hall, J. W. 2000. Reproducibility of enzyme-linked immunosorbent assay and immunofluorescence for detecting Clavibacter michiganensis subsp. sepedonicus in multiple laboratories. Bull. OEPP 30:397-401.

5. Dickey, R. S. 1979. Erwinia chrysanthemi: A comparative study of phenotypic properties of strains from several hosts and other Erwinia species. Phytopathology 69:324-329.

6. Dickey, R. S., Zumoff, C. H., and Uyemoto, J. K. 1984. Erwinia chrysanthemi: Serological relationships among strains from several hosts. Phytopathology 74:1388-1394.

7. Ehrmann, M., Ludwig, W., and Schleifer, K. H. 1994. Reverse dot blot hybridization: A useful method for the direct identification of lactic acid bacteria in fermented food. FEMS Microbiol. Lett. 117:143-149.

8. Elphinstone, J. G., Stead, D. E., Caffier, D., Janse, J. D., Lopez, M. M., Mazzucchi, U., Muller, P., Persson, P., Rauscher, E., Schiessendoppler, E., Santos, M. S., Stefani, E., and van Vaerenbergh, J. 2000. Standardization of methods for detection of Ralstonia solanacearum in potato. Bull. OEPP 30:391-395.

9. Fessehaie, A., De Boer, S. H., and Lévesque, C. A. 2002. Molecular 
characterization of DNA encoding 16S-23S rRNA intergenic spacer regions and 16S rRNA of Erwinia species. Can. J. Microbiol. 48:387-398.

10. Fessehaie, A., De Boer, S. H., Quail, A., and Lévesque, C. A. 2001. Development of a DNA array for identification and detection of pathogenic bacteria associated with potato. Pages 389-392 in: Plant Pathogenic Bacteria. S. H. De Boer, ed. Kluwer Academic Publishers, Dordrecht, The Netherlands.

11. Godovikova, T. S., Orlova, T. N., Dobrikova, E. I., Shamanin, V. A., Zarytova, V. F., Vorob'eva, N. V., Serdiukova, N. A., Shamanina, M. I., Petruseva, I. O., and Pitsenko, N. D. 1994. Highly-sensitive nonradioactive detection of the tick-borne encephalitis virus. Bioorg. Khim. 20:1196-1205.

12. Gutell, R. R., and Fox, G. E. 1988. A compilation of large subunit RNA sequences presented in a structural format. Nucleic Acids Res. 16(Suppl.):r175-r201.

13. Hamels, S., Gala, J. L., Dufour, S., Vannuffel, P., Zammatteo, N., and Remacle, J. 2001. Consensus PCR and microarray for diagnosis of the genus Staphylococcus, species, and methicillin resistance. Biotechniques 31:1364-1372.

14. Janse, J. D. 1996. Potato brown rot in western Europe. History, present occurrence and some remarks on possible origin, epidemiology and control strategies. Bull. OEPP 26:679-695.

15. Janse, J. D., and Ruissen, M. A. 1988. Characterization and classification of Erwinia chrysanthemi strains from several hosts in The Netherlands. Phytopathology 78:800-808.

16. Jeng, R. S., Svircev, A. M., Myers, A. L., Beliaeva, L., Hunter, D. M., and Hubbes, M. 2001. The use of $16 \mathrm{~S}$ and $16 \mathrm{~S}-23 \mathrm{~S}$ rDNA to easily detect and differentiate common gram-negative orchard epiphytes. J. Microbiol. Methods 44:69-77.

17. Jensen, M. A., Webster, J. A., and Straus, N. 1993. Rapid identification of bacteria on the basis of polymerase chain reaction-amplified ribosomal DNA spacer polymorphisms. Appl. Environ. Microbiol. 59:945-952.

18. Kawasaki, E. S., and Chehab, F. F. 1994. Analysis of gene sequences by hybridization of PCR-amplified DNA to covalently bound oligonucleotide probes. The reverse dot blot method. Methods Mol. Biol. 28:225236.

19. Lane, D. 1991. 16S/23S sequencing. Pages 115-175 in: Nucleic Acid Techniques in Bacterial Systematics. E. Stackebrandt and $M$. Goodfellow, eds. John Wiley \& Sons, Chichester, England.

20. Lévesque, C. A. 1997. Molecular detection tools in integrated disease management: Overcoming current limitations. Phytoparasitica 25:3-7.

21. Lévesque, C. A. 2001. Molecular methods for detection of plant pathogens-What is the future? Can. J. Plant Pathol. 24:333-336.

22. Lévesque, C. A., Harlton, C. E., and de Cock, A. W. A. M. 1998. Identification of some oomycetes by reverse dot blot hybridization. Phytopathology 88:213-222.

23. Li, X., and De Boer, S. H. 1995. Comparison of 16 S ribosomal RNA genes in Clavibacter michiganensis subspecies with other coryneform bacteria. Can. J. Microbiol. 41:925-929.

24. Li, X., and De Boer, S. H. 1995. Selection of polymerase chain reaction primers from an RNA intergenic spacer region for specific detection of Clavibacter michiganensis subsp. sepedonicus. Phytopathology 85: 837-842.

25. Louws, F., Rademaker, J., and de Bruijn, F. 1999. The three Ds of PCR- based genomic analysis of phytobacteria: Diversity, detection, and disease diagnosis. Annu. Rev. Phytopathol. 37:81-125.

26. Martin, C., Roberts, D., van Der Weide, M., Rossau, R., Jannes, G., Smith, T., and Maher, M. 2000. Development of a PCR-based line probe assay for identification of fungal pathogens. J. Clin. Microbiol. 38:37353742.

27. McGuire, R. G., and Kelman, A. 1984. Reduced severity of Erwinia soft rot in potato tubers with increased calcium content. Phytopathology 74:1250-1256

28. Pérombelon, M. C. M., Bertheau, Y., Cambra, M., Frechon, D., Lopez, M. M., Niepold, F., Persson, P., Sletten, A., Toth, I. K., van Vuurde, J. W. L., and van der Wolf, J. M. 1998. Microbiological, immunological and molecular methods suitable for commercial detection and quantification of the blackleg pathogen, Erwinia carotovora subsp. atroseptica, on seed potato tubers: A review. Bull. OEPP 28:141-155.

29. Pérombelon, M. C. M., and Kelman, A. 1980. Ecology of the soft rot erwinias. Annu. Rev. Phytopathol. 18:361-387.

30. Schaad, N. W., Berthier, S. Y., Sechler, A., and Knorr, D. 1999. Detection of Clavibacter michiganensis subsp. sepedonicus in potato tubers by BIO-PCR and an automated real-time fluorescence detection system. Plant Dis. 83:1095-1100.

31. Seifarth, W., Krause, U., Hohenadl, C., Baust, C., Hehlmann, R., and Leib-Mosch, C. 2000. Rapid identification of all known retroviral reverse transcriptase sequences with a novel versatile detection assay. AIDS Res. Hum. Retrovir. 16:721-729.

32. Smid, E. J., Hansen, A. H. J., and Gorris, L. G. M. 1995. Detection of Erwinia carotovora subsp. atroseptica and Erwinia chrysanthemi in potato tubers using polymerase chain reaction. Plant Pathol. 44:10581069.

33. Toth, I. K., Avrova, A. O., and Hyman, L. J. 2001. Rapid identification and differentiation of the soft rot erwinias by $16 \mathrm{~S}-23 \mathrm{~S}$ intergenic transcribed spacer-PCR and restriction fragment length polymorphism analyses. Appl. Environ. Microbiol. 67:4070-4076.

34. Uehara, T., Kushida, A., and Momota, Y. 1999. Rapid and sensitive identification of Pratylenchus spp. using reverse dot blot hybridization. Nematology 1:549-555.

35. Van der Wolf, J. M., Bonants, P. J. M., Smith, J. J., Hagenaar, M., Nijhuis, E., Van Beckhoven, J. R. C. M., Saddler, G. S., Trigalet, A., and Feuillade, R. 1998. Genetic diversity of Ralstonia solanacearum race 3 in Western Europe determined by AFLP, RC-PFGE and Rep-PCR. Pages 44-49 in: Bacterial Wilt Disease: Molecular and Ecological Aspects. P. Prior, C. Allen, and J. Elphinstone, eds. Springer-Verlag, Berlin.

36. Wu, L., Thompson, D. K., Li, G., Hurt, R. A., Tiedje, J. M., and Zhou, J. 2001. Development and evaluation of functional gene arrays for detection of selected genes in the environment. Appl. Environ. Microbiol. 67:5780-5790.

37. Yang, Y., Corley, N., and Garcia-Heras, J. 2001. Reverse dot-blot hybridization as an improved tool for the molecular diagnosis of point mutations in congenital adrenal hyperplasia caused by 21-hydroxylase deficiency. Mol. Diagn. 6:193-199.

38. Ye, F., Li, M. S., Taylor, J. D., Nguyen, Q., Colton, H. M., Casey, W. M., Wagner, M., Weiner, M. P., and Chen, J. 2001. Fluorescent microspherebased readout technology for multiplexed human single nucleotide polymorphism analysis and bacterial identification. Hum. Mutat. 17:305-316. 